\title{
Wnt-11 promotes neuroendocrine-like differentiation, survival and migration of prostate cancer cells
}

\author{
Pinar Uysal-Onganer ${ }^{1 *}$, Yoshiaki Kawano ${ }^{1}$, Mercedes Caro ${ }^{3}$, Marjorie M Walker², Soraya Diez ${ }^{1}$, \\ R Siobhan Darrington', Jonathan Waxman', Robert M Kypta1,3*
}

\begin{abstract}
Background: Wnt-11 is a secreted protein that modulates cell growth, differentiation and morphogenesis during development. We previously reported that Wnt-11 expression is elevated in hormone-independent prostate cancer and that the progression of prostate cancer from androgen-dependent to androgen-independent proliferation correlates with a loss of mutual inhibition between Wnt-11- and androgen receptor-dependent signals. However, the prevalence of increased expression of Wnt-11 in patient tumours and the functions of Wnt-11 in prostate cancer cells were not known.
\end{abstract}

Results: Wnt-11 protein levels in prostate tumours were determined by immunohistochemical analysis of prostate tumour tissue arrays. Wnt-11 protein was elevated in 77/117 of tumours when compared with 27 benign prostatic hypertrophy specimens and was present in $4 / 4$ bone metastases. In addition, there was a positive correlation between Wnt-11 expression and PSA levels above $10 \mathrm{ng} / \mathrm{ml}$. Androgen-depleted LNCaP prostate cancer cells form neurites and express genes associated with neuroendocrine-like differentiation (NED), a feature of prostate tumours that have a poor prognosis. Since androgen-depletion increases expression of Wnt-11, we examined the role of Wnt-11 in NED. Ectopic expression of Wnt-11 induced expression of NSE and ASCL1, which are markers of NED, and this was prevented by inhibitors of cyclic AMP-dependent protein kinase, consistent with the known role of this kinase in NED. In contrast, Wnt-11 did not induce NSE expression in RWPE-1 cells, which are derived from benign prostate, suggesting that the role of Wnt-11 in NED is specific to prostate cancer. In addition, silencing of Wnt-11 expression in androgen-depleted LNCaP cells prevented NED and resulted in apoptosis. Silencing of Wnt11 gene expression in androgen-independent PC3 cells also reduced expression of NSE and increased apoptosis. Finally, silencing of Wnt-11 reduced PC3 cell migration and ectopic expression of Wnt-11 promoted LNCaP cell invasion.

Conclusions: These observations suggest that the increased level of Wnt-11 found in prostate cancer contributes to tumour progression by promoting NED, tumour cell survival and cell migration/invasion, and may provide an opportunity for novel therapy in prostate cancer.

\section{Background}

Wnt genes code for secreted signalling proteins that are responsible for the development and repair of many organs in the body and aberrant activation of Wnt signalling is implicated in tumorigenesis [1]. We have previously reported increased expression of Wnt-11 mRNA in androgen-independent prostate cancer (PCa) [2].

\footnotetext{
* Correspondence: p.onganer@imperial.ac.uk; r.kypta@ic.ac.uk

'Department of Oncology, Imperial College London, London, UK
}

Wnt-11 is best known for its role during development, for example, it is required for convergent extension movements during gastrulation [3] and kidney morphogenesis [4]. In addition, cell-based assays have demonstrated that Wnt-11 promotes cardiac differentiation [5], increases proliferation, migration and transformation of intestinal epithelial cells [6], reduces apoptosis in breast cancer cells [7] and increases cell viability in chinese hamster ovary $(\mathrm{CHO})$ cells [8]. The signals downstream of Wnt-11 are not fully characterised. Wnt-11 has been 
reported to inhibit JNK and NF-kappaB [8], activate PKC and JNK [9] and activate cAMP response element binding protein (CREB) family members [10]. Wnt-11 does not appear to stabilise $\beta$-catenin and is frequently found to inhibit 'canonical' Wnt/ $\beta$-catenin signalling $[2,8,11]$.

Despite its high level of expression in androgen-independent PCa cells, ectopic expression of Wnt-11 inhibits the growth of the androgen-dependent LNCaP cells [2]. The morphology of Wnt-11-transfected LNCaP cells resembles that of androgen-depleted LNCaP cells as they undergo neuroendocrine-like differentiation (NED) [12], which we previously reported induces expression of endogenous Wnt-11 [2]. This raised the possibility that Wnt-11 may promote NED. Neuroendocrine (NE) cells constitute a minor cell population in the normal prostate that is thought to regulate prostatic growth and differentiation. However, in prostate tumours, the number of NE-like cells correlates with tumour progression, poor prognosis and the androgen-independent state $[12,13]$. These and other observations have led to the suggestion that $\mathrm{PCa}$ cells transdifferentiate to become NE-like. Agents that induce NED elevate intracellular levels of cyclic AMP (cAMP), and it has been postulated that cAMP-mediated signalling is a primary pathway of NED in vivo [14]. Here, we have further investigated the function of Wnt-11 and show that it promotes NED in a PKA-dependent manner and promotes prostate cancer cell survival, migration and invasion.

\section{Results}

\section{Increased expression of Wnt-11 in prostate tumours}

In order to determine whether Wnt-11 protein levels are elevated in patient tumours, we used anti-Wnt-11 antibodies to localise Wnt-11 expression in sections taken from human prostate and prostate tumour tissue (Figure $1)$. Benign prostate sections (27 cases) were either negative (not shown) or exhibited weak expression of Wnt11 in luminal epithelial cells and in some smooth muscle cells (Figure 1a). Malignant prostate showed stronger expression of Wnt-11 in luminal epithelial cells (Figures $1 \mathrm{~b}, \mathrm{c})$. Immunohistochemical analysis of Wnt-11 in tumour tissue arrays indicated that the level of Wnt-11 was elevated in 77/117 (66\%) of tumours (Figures 1b $1 d$ ), with particularly strong staining in 28/117 (24\%) of cases (Figure 1f). In addition, Wnt-11 was detected in 2/ 2 examples of perineural invasion (Figure 1e) and in 4/4 bone metastases (Figure 1g, high expression of the androgen receptor (AR) is shown in an adjacent section) suggesting a possible role for Wnt-11 in invasion and/or metastasis. Finally, Wnt-11 and AR were found to be co-expressed in prostate tumour cells (Figures $1 \mathrm{i}, \mathrm{j}$ ). The level of Wnt-11 expression was generally higher in $\mathrm{PCa}$ compared to benign prostate. Analysis in relation to conventional prognostic indices of $\mathrm{PCa}$ showed a negative correlation with Gleason grade; Wnt-11 was more frequently found in Gleason 3 tumours than in Gleason 4 tumours and in Gleason $<8$ compared to Gleason > 8 , suggesting that Wnt-11 is not a general marker for tumour dedifferentiation. However, Wnt-11 was significantly elevated in tumours from patients with PSA levels above $10 \mathrm{ng} / \mathrm{ml}$ (Table 1).

\section{Wnt-11 promotes neuroendocrine-like differentiation in prostate cancer cells}

We noted that the morphology of Wnt-11-transfected LNCaP cells resembled that of androgen-depleted LNCaP cells as they undergo NED, raising the possibility that Wnt-11 may be involved in NED. To test this possibility, we examined the effects of downregulation of endogenous Wnt-11 in LNCaP cells. LNCaP cell lines were generated expressing WNT11 shRNA or a nonsilencing control shRNA. RT-PCR analysis confirmed upregulation of Wnt-11 upon hormone-depletion in control shRNA cells but not in WNT11 shRNA cells (Figure 2a). Culture of control shRNA cells in hormonedepleted medium for a longer period of time resulted in growth arrest and generation of neurite-like processes (Figure 2b), as previously described for parental LNCaP cells $[15,16]$. In contrast, hormone-depletion of WNT11 shRNA cells produced an initial morphological response after one day in hormone-depleted medium (Figure 2b), but thereafter the number of neuritic processes decreased, with a significant reduction in the percentage of cells with neurites after 4 days (Figure 2c). After 18 days in hormone-depleted medium, control shRNA cells remained viable and had long neurites, whereas almost all the WNT11 shRNA cells had died (Figure 2b).

To determine if the differences in neurite outgrowth resulted from an inability of WNT11 shRNA cells to undergo NED, extracts from cells cultured in hormonedepleted medium were probed for a marker of NED, neuron-specific enolase (NSE) (Figure 3a). Control shRNA cells cultured in hormone-depleted medium expressed NSE, whereas hormone-depleted WNT11 shRNA expressed very little NSE. Thus, endogenous Wnt-11 appears to be required for expression of NSE, a marker for NED, in hormone-depleted LNCaP cells.

Ectopic expression of Wnt-11 reduces proliferation of LNCaP cells [2] and cell lines overexpressing Wnt-11 could be maintained for only a limited number of passages, suggesting that Wnt-11 induces LNCaP cell growth arrest or differentiation. To determine whether increased expression of Wnt-11 is sufficient to promote NED, LNCaP cells were transfected with a Wnt-11 expression vector and the levels of expression of NSE and a second gene, $A S C L 1$, whose expression is increased during NED [17] were measured (Figure $3 \mathrm{~b}$ and 


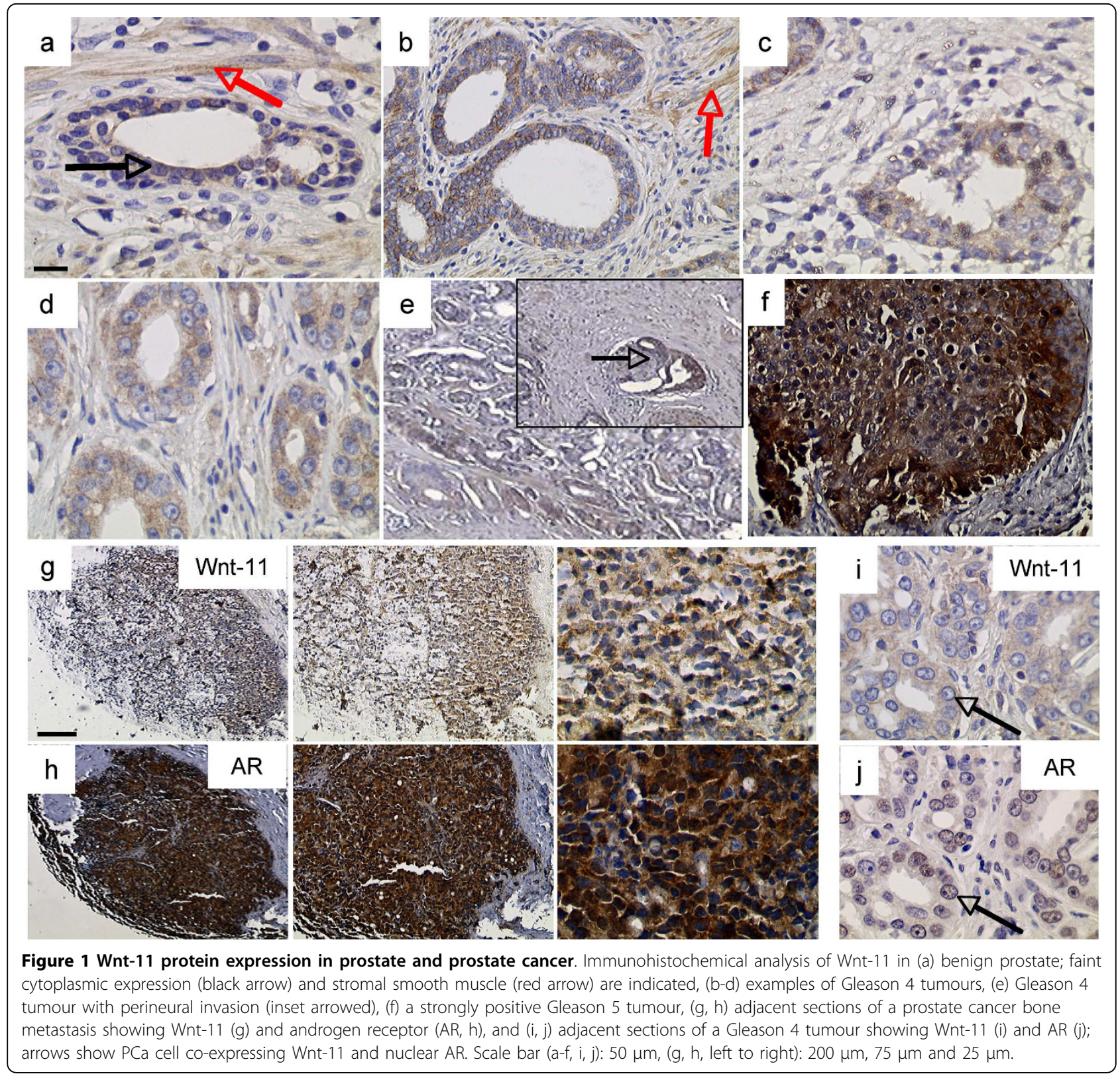

Additional File 1). Wnt-11 increased expression of NSE and $A S C L 1$, suggesting that Wnt-11 is sufficient to induce NED in LNCaP cells. Androgen depletion activates NED in LNCaP cells by increasing cellular levels of cAMP, leading to activation of PKA [15]. Therefore we tested the effect of inhibiting cAMP signalling on Wnt11 induction of these same genes. Wnt-11 induction of $N E S$ and $A S C L 1$ was reduced to baseline levels upon treatment with four independent PKA inhibitors (H89, KT5720, Rp-cAMPS and PKI, Figure 3c), suggesting that Wnt-11 induction of NED requires activation of PKA.

NE-like differentiation is associated with a loss of AR expression in both LNCaP cells and human tumours
[13]. Since Wnt-11 inhibits AR activity, we determined whether ectopic expression of Wnt-11 in LNCaP cells affected the expression level or localisation of AR. The level of AR expression was reduced in LNCaP cells expressing Wnt-11, but its subcellular localisation was not affected (Figure 3d). This suggests that Wnt-11 induction of NE-like differentiation in LNCaP cells might result from its ability to reduce AR expression. However, LNCaP cells expressing Wnt-11 still expressed some AR. In order to test whether Wnt-11 might also play a role in NED in AR-negative PCa cells, we conducted experiments using PC3 cells, an AR-negative, PCa cell line that expresses high levels of Wnt-11 [2] 
Table 1 Comparison of Wnt-11 protein expression and tumour stage

\begin{tabular}{ll}
\hline $\begin{array}{l}\text { Comparison } \\
\text { (Wnt-11 -ve versus Wnt-11 +ve) }\end{array}$ & $\begin{array}{l}\boldsymbol{p} \text { value } \\
\text { (Chi-squared test) }\end{array}$ \\
\hline PSA $<10 \mathrm{ng} / \mathrm{ml}$ versus PSA > 10 ng/ml & 0.0001 \\
Gleason 4 versus Gleason 3 & 0.005 \\
Gleason 5 versus Gleason 4 & 0.3 \\
Gleason sum 7 versus Gleason sum 6 & 0.01 \\
Gleason sum >8 versus Gleason sum <8 & 0.02 \\
PNI +ve versus PNI -ve & 0.6 \\
\hline
\end{tabular}

Tumour samples from 117 patients were analysed. 77/117 (66\%) of tumours contained Wnt-11-positive epithelial cells, 28/117 (24\%) of tumours were strongly Wnt- 11 positive and $4 / 4$ bone metastases were Wnt- 11 positive. Wnt11 expression correlated with increased serum PSA but lower Gleason score. Statistical significance was determined using chi-squared test and results were considered significant for $\mathrm{p}<0.05$. PSA, prostate-specific antigen; PNI, perineural invasion.

and displays some characteristics of NE cells [13]. It was not possible to generate PC3 cell lines stably expressing shRNAs and the transfection efficiency of the shRNA vectors was too low to achieve silencing of WNT11. Therefore, WNT11 was silenced by transient transfection of siRNA oligonucleotides. Silencing of WNT11 in PC3 cells resulted in a reduction of NSE mRNA levels $24 \mathrm{~h}$ after transfection (Figure 3e). These results suggest that Wnt-11 plays a role in NED of both AR-positive and AR-negative prostate cancer cells. In order to determine if Wnt-11 was also involved in NED in untransformed prostate epithelial cells, we expressed Wnt-11 in RWPE-1 cells, which are derived from normal prostate and exhibit features of prostate progenitor cells, including the ability to differentiate into NE cells [18]. Expression of Wnt-11 did not affect the level of NSE mRNA in RWPE-1 cells (Figure 3f). This difference did not result from differences in the amount of Wnt-11 protein expressed, since transfection efficiency was similar in LNCaP and RWPE-1 cells (Additional File 2). This suggests that Wnt-11 promotes NED in PCa cells but not in untransformed prostate epithelial cells.

\section{Silencing of WNT11 increases apoptosis in prostate cancer cells}

Silencing of WNT11 appeared to reduce the viability of $\mathrm{LNCaP}$ cells grown in androgen-depleted medium (Figure 2b). In order to determine if this reflected an effect on apoptosis, cell extracts from androgen-depleted control and WNT11 shRNA cells were probed for cleaved PARP and cleaved caspase 9, which are frequently used as indicators of apoptosis. The levels of

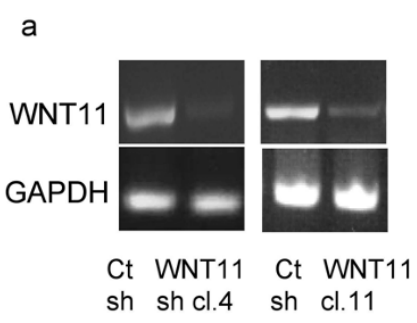

C
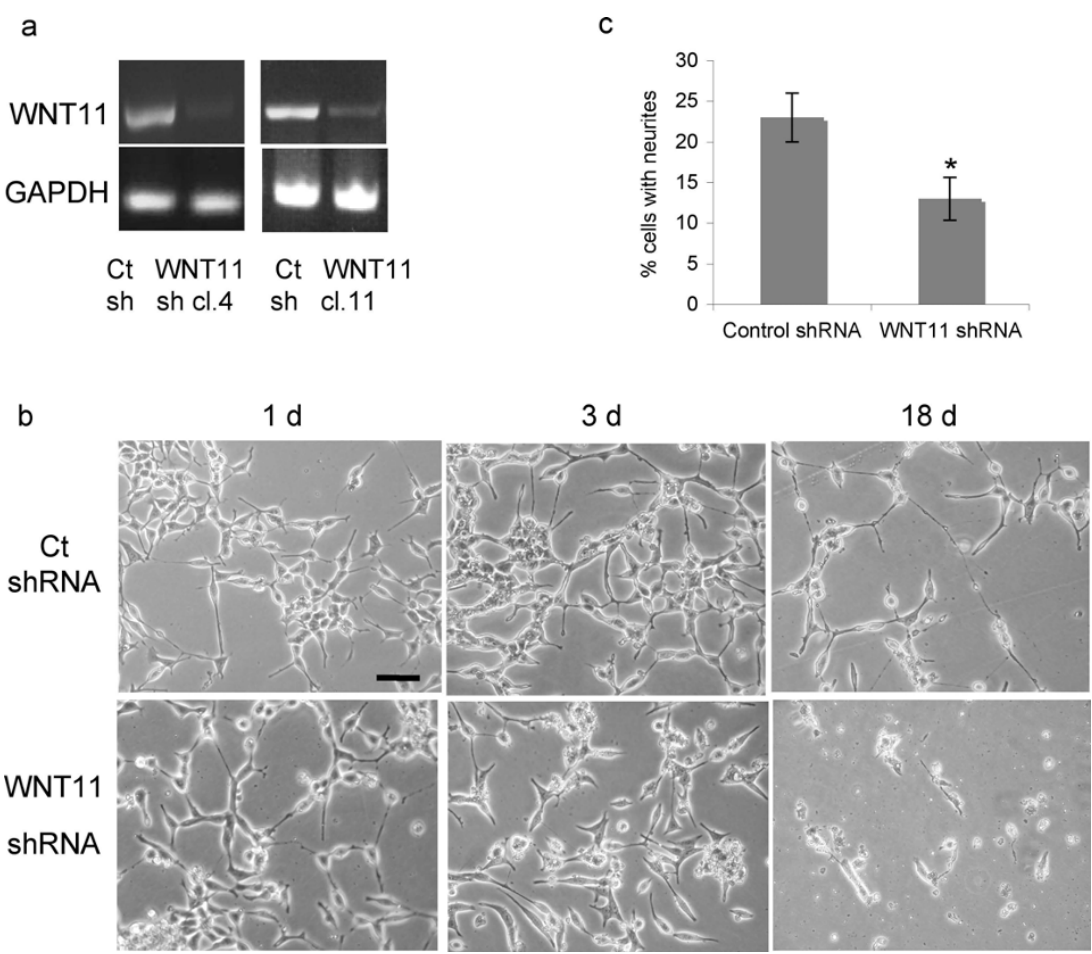

Figure 2 Silencing of Wnt-11 reduces survival of androgen-depleted LNCP cells. (a) RT-PCR of WNT11 and GAPDH in LNCaP cell lines stably expressing control (Ct) shRNA or WNT11 shRNA (clones 4 and 11) cultured for 7 days in hormone-depleted medium. (b) Images of control shRNA or WNT11 shRNA (clone 11) LNCaP cells cultured for the indicated times in hormone-depleted medium. Scale bar $200 \mu \mathrm{m}$. Similar results to those shown were obtained using three independent WNT11 shRNA clones. (c) Quantitation of neurite outgrowth of control shRNA or WNT11 shRNA (clone 11) LNCaP cells cultured for 4 days in hormone-depleted medium. The data presented are the means (+/- SD) derived from counting three fields of 100 cells; ${ }^{*} p<0.05$. 


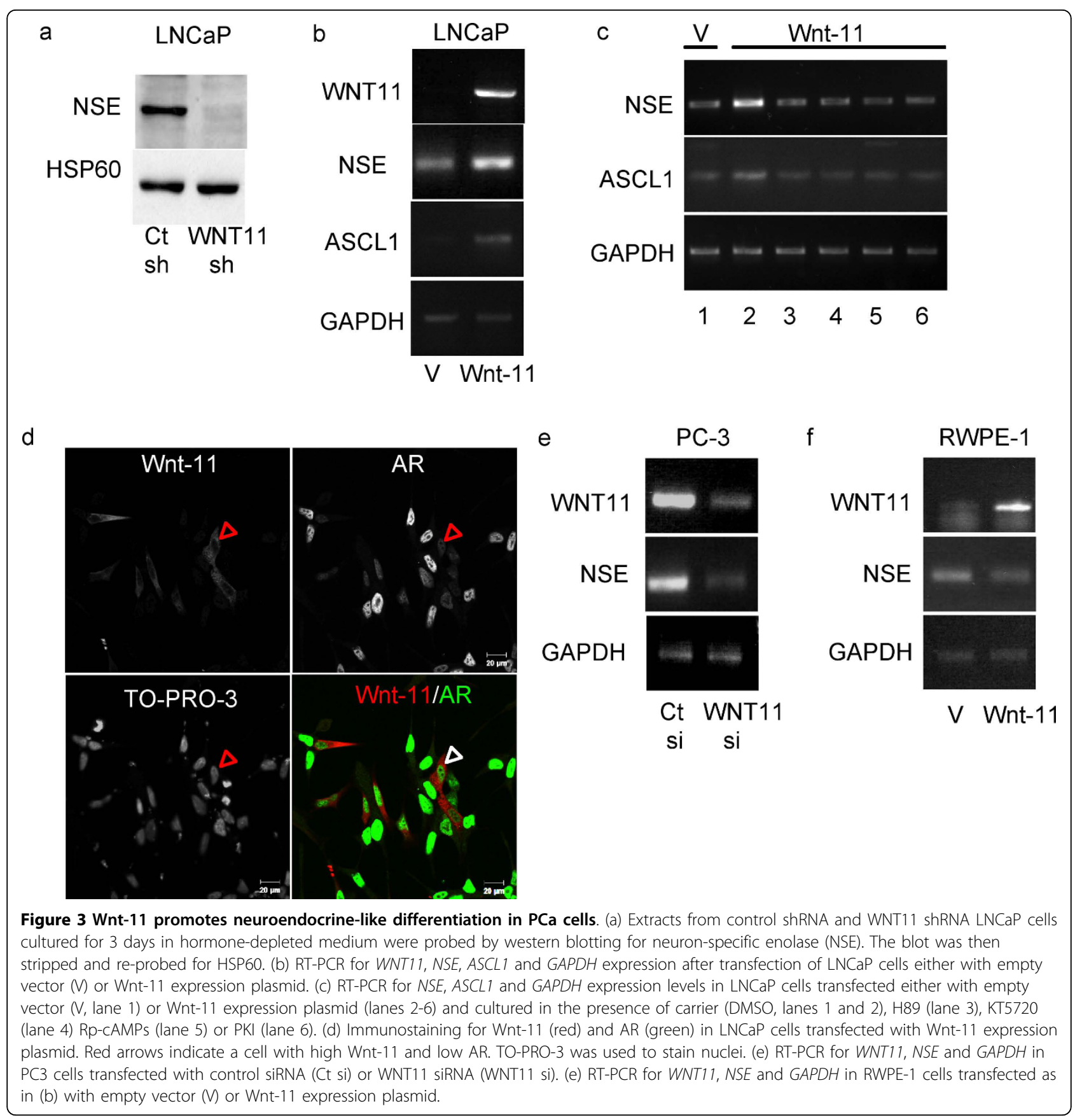

both cleaved proteins were elevated in androgendepleted WNT11 shRNA cells when compared to controls (Figure 4a). Furthermore, caspase assays conducted using extracts from WNT11 shRNA cells and control shRNA cells grown in androgen-depleted medium confirmed that loss of Wnt-11 increases apoptosis in androgen-depleted cells (Figure 4b). To determine whether endogenous Wnt-11 also protected PC3 cells from apoptosis, PC3 cells were transfected with control or WNT11 siRNA oligonucleotides and then assayed for caspase activity. Caspase activity was higher in WNT11 siRNA cells compared to in control cells (Figure 4c), indicating that endogenous Wnt-11 also plays a role in preventing apoptosis in this androgen-independent $\mathrm{PCa}$ cell line.

Wnt-11 promotes prostate cancer cell migration and invasion

Wnt-11 has been reported to affect migration during development and in some cell lines $[6,19,20]$. To 


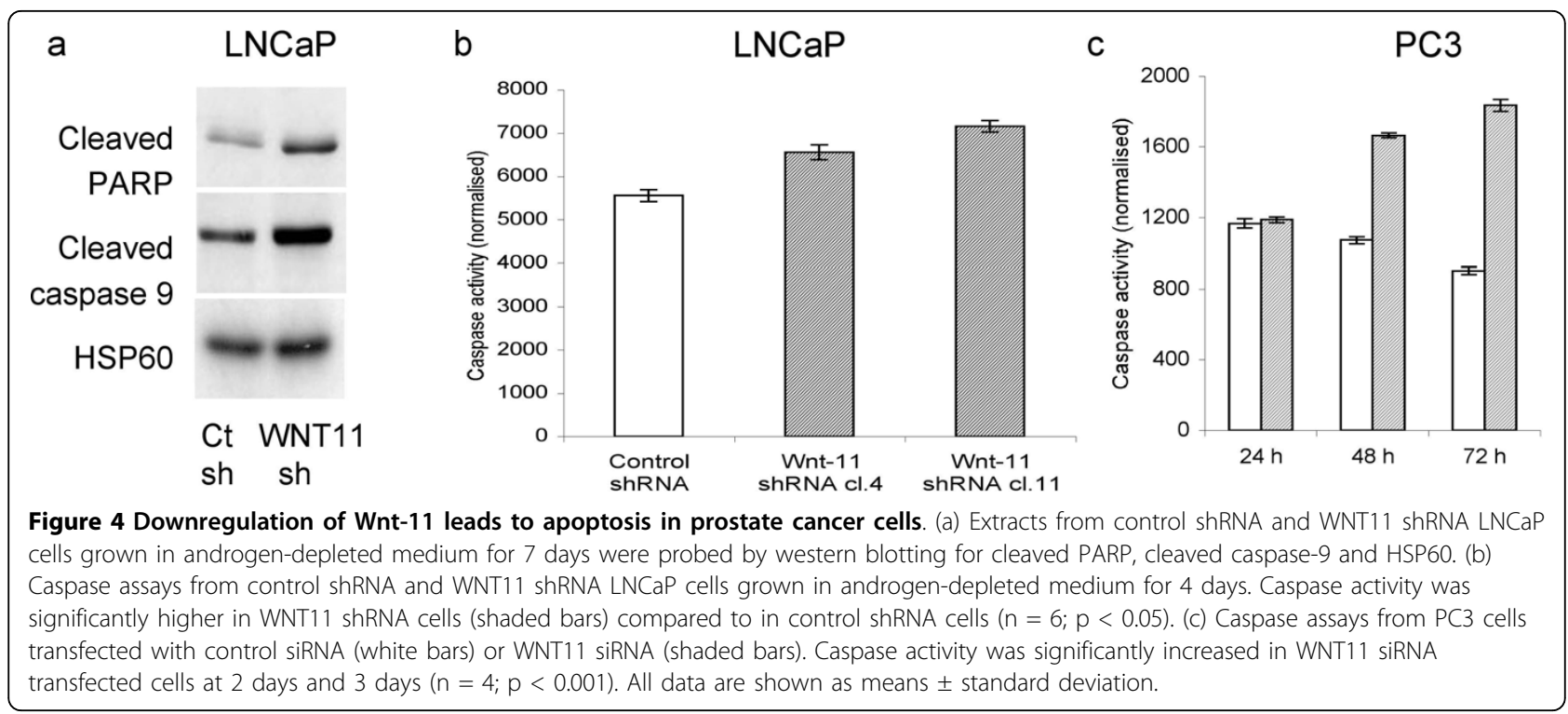

determine if Wnt-11 was involved in PC-3 cell migration, cells were transfected with control or WNT11 siRNA oligonucleotides and assayed for their ability to migrate through Transwell filters (Figure 5a). Compared to the control siRNA, WNT11 siRNA reduced PC3 cell transverse migration, suggesting that endogenous Wnt11 also plays a role in migration of this metastatic $\mathrm{PCa}$ cell line. LNCaP cells, in contrast to PC3 cells, do not migrate and are not normally invasive. However, LNCaP cells can be induced to invade by secreted factors, for example, neurotensin and components of FCS [21]. In order to determine if Wnt-11 overexpression promotes invasion, $\mathrm{LNCaP}$ cells were transfected with vector or Wnt-11 expression plasmids and, after two days to allow for Wnt-11 expression, plated on Matrigel-coated filters in the presence of $1 \%$ FCS and invasion assays performed. Expression of Wnt-11 increased the number of invading LNCaP cells compared to control cells (Figure $5 \mathrm{~b}$ ). MTT assays indicated that there were no differences in the total numbers of cells during the assay (data not shown). Together, these results indicate that Wnt-11 not only plays roles in NED and survival of prostate cancer cells, but also in their ability to migrate and invade.

\section{Discussion}

Our results suggest that Wnt-11 can endow prostate tumour cells with three properties important for $\mathrm{PCa}$ progression, namely, increased resistance to apoptosis, neuroendocrine-like differentiation and increased motility. The increased resistance to apoptosis provides a survival mechanism for $\mathrm{PCa}$ cells not only under
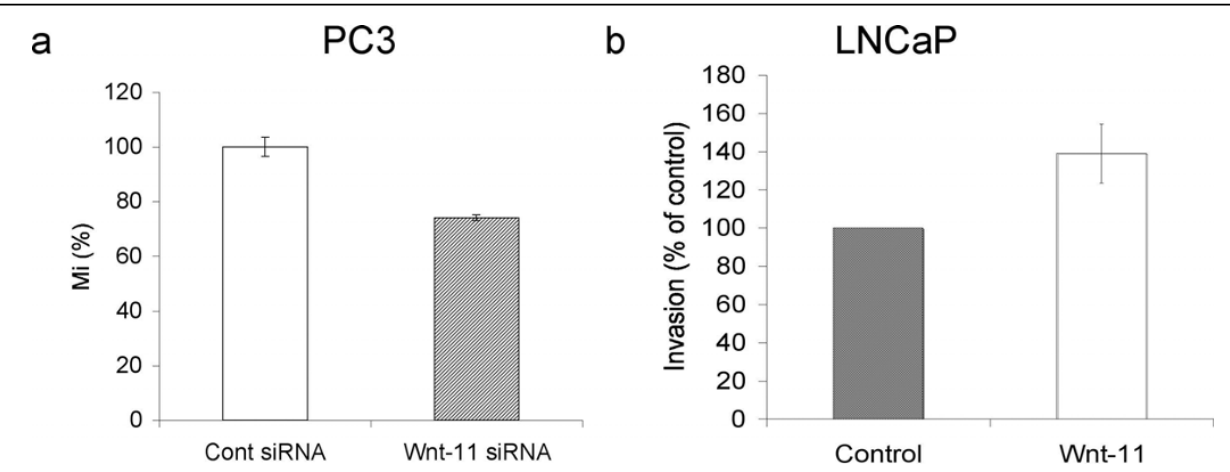

Figure 5 Wnt-11 promotes prostate cancer cell migration and invasion. (a) PC3 cells were transfected for 72 hours with control or WNT11 siRNAs, plated on Transwell filters and the extent of migration determined. WNT11 siRNA decreased transverse migration $(p=0.02 ; n=9)$. Results are plotted as Migration Index (Mi), which is the percentage of migrated cells compared to the total number of cells seeded. (b) LNCaP cells were transfected with empty vector or Wnt-11 expression plasmid, plated on Matrigel-coated transwell filters and the extent of invasion determined. The extent of invasion was normalised to that in control cells $(p=0.01 ; n=5)$. The total cell number did not change during the course of the experiment. 
conditions of hormone-depletion but also in castrateresistant PCa. Wnt-11 has previously been shown to be important for the survival and/or viability of MCF-7 breast cancer cells [7] and $\mathrm{CHO}$ cells [8], suggesting that this is a general role for Wnt-11. It is not clear whether the survival function of Wnt-11 in PCa is direct or results from its effects on NED. WNT11 siRNA reduced NSE expression in PC3 cells after $24 \mathrm{~h}$, before it significantly increased caspase activity (48 h), suggesting that the principal role of Wnt-11 is to maintain NED and that the inability to do this leads to apoptosis. This would also be consistent with the known link between NED and resistance to apoptosis [22]. Wnt-11 is known to play a role in differentiation in several tissues, for example, promoting cardiomyogenic differentiation of mesodermal cells [23], mouse embryonic stem cells [24], endothelial circulating progenitor cells [25] and bone marrow mononuclear cells [9], and this occurs without effects on cell proliferation or survival in the latter two examples. Nevertheless, Wnt-11 could play an independent role in PCa cell survival and NED.

Wnt-11 inhibits AR transcriptional activity in LNCaP cells and NED is associated with a loss of AR expression [13]. Since ectopic expression of Wnt-11 in LNCaP cells reduced the level of AR (Figure 3d), it is possible that Wnt-11 induces NED indirectly by reducing AR expression. However, in contrast to what was observed in LNCaP-derived NE-like cell lines, which express no AR [13], expression of Wnt-11 did not lead to a complete loss of AR. Moreover, Wnt-11 and AR are co-expressed in other prostate cancer cell lines [2] and in prostate tumour cells (Figure 1i, j). Wnt-11 induction of NED was inhibited by four different inhibitors of PKA, suggesting that Wnt-11 activates activate PKA to induce NED. The signalling events that take place downstream of Wnt-11 during NED are not known, but they may involve activation of CREB, as observed during heart morphogenesis [10], since CREB is activated upon induction of NED and constitutively-active CREB can itself induce NED [26].

The role of Wnt-11 in NED appears to be restricted to PCa cells since ectopic expression of Wnt-11 did not increase expression of NSE in non-tumorigenic RWPE-1 cells, even though these cells have the potential to express this gene [18]. NE cells in the human prostate are thought to originate from a stem/progenitor cell that also gives rise to basal and secretory epithelial cells [27]. They can be distinguished from most NE-like cells derived from PCa cells because they are terminally differentiated and do not co-express luminal epithelial cell markers such as AR [13]. A recent study provided evidence that cells initiating PCa are derived from a malignantly transformed 'intermediate' cell that acquires stem cell-like activity, rather than from a transformed normal prostate stem cell [28]. If this holds true, Wnt-11 might specifically promote NED of PCa cells but not of normal progenitors, which could be important when considering Wnt-11 as a potential target for therapy.

A role for Wnt-11 in cell migration was first suggested by studies in Xenopus and zebrafish, where Wnt-11 promotes convergent extension movements during gastrulation $[19,20]$. More recently, Wnt-11 was shown to promote migration of intestinal epithelial cells [6]. Our demonstration that Wnt-11 is required for migration of PC3 cells and promotes invasion of LNCaP cells (Figure 5) underscores the potential of this molecule to promote $\mathrm{PCa}$ metastasis. Consistent with this possibility, Wnt-11 was detected in PCa cells in the process of invading the neural sheath (Figure 1d) and in PCa bone metastases (Figure 1g).

The level of Wnt-11 expression was higher in PCa compared to benign prostate but correlated inversely with Gleason grade. This was unexpected given the high level of expression of Wnt-11 in some high-grade tumours (Figure 1f) as well as in all prostate tumour bone metastases examined (Figure $1 \mathrm{~g}$ and Table 1). Wnt-11 may play a role in metastasis independent of the state of differentiation or have different functions in low grade and high-grade PCa. For example, in lowgrade PCa Wnt-11 might prevent apoptosis, whereas in high-grade $\mathrm{PCa}$ it could promote invasion and, through its effects on NED, promote progression to androgenindependence. The relative importance of these roles at each cancer stage and in each patient would likely depend on other mutations present in the tumour. Interestingly, there was a positive correlation between Wnt-11 expression and patient PSA levels above $10 \mathrm{ng} /$ ml. This might seem at odds with the fact that Wnt-11 reduces AR activity in $\mathrm{LNCaP}$ cells. However, we previously showed that Wnt-11 does not inhibit AR activity in androgen-independent AR-positive prostate cancer cell lines [2]. Therefore it is possible that the Wnt-11positive tumours from patients with high PSA levels contain an androgen-independent population of tumour cells. A more detailed analysis using a larger cohort of patients with follow-on data will be required to test these possibilities.

\section{Conclusions}

This study shows that Wnt-11 protein levels are elevated in a significant proportion of human prostate tumours. In addition, it demonstrates that Wnt-11 is both necessary and sufficient for induction of neuroendocrine-like differentiation in prostate cancer cells and that this requires PKA activity. Finally, it shows that Wnt-11 is required for prostate cancer cell survival and promotes prostate cancer cell invasion. Taken together, these observations suggest that Wnt-11 is a potential therapeutic target in the treatment of prostate cancer. 


\section{Methods}

\section{Cell culture}

LNCaP, PC3 and RWPE-1 cells were from the American Type Culture Collection (Rockville, MD, USA), purchased through LGC Promochem. Cell stocks were frozen within six months of receipt and, upon thawing, used for up to six months. LNCaP and PC3 cells were grown at $37^{\circ} \mathrm{C}, 5 \% \mathrm{CO}_{2}$ in $\mathrm{RPMI}-1640$ (Invitrogen) with 10\% foetal bovine serum (FBS; First Link, UK) and antibiotics (100 units/mL penicillin, $100 \mu \mathrm{g} / \mathrm{mL}$ streptomycin, Invitrogen, UK). RWPE-1 cells were grown in Keratinocyte serum-free medium (KSFM) supplemented with bovine pituitary extract and EGF (Invitrogen, UK). LNCaP shRNA clones were grown in RPMI with $10 \%$ tetracycline-free FBS (Clontech, Takara Bio USA). For hormone-depletion experiments, LNCaP cells were cultured in phenol red-free RPMI containing 5\% charcoalstripped serum (CSS, First Link, UK). For transfections, cells were cultured in Opti-MEM (Invitrogen). The following PKA inhibitors were used: H89 (10 $\mu \mathrm{M}$, Calbiochem, UK), KT5720 (5 $\mu \mathrm{M}$, Calbiochem, UK), RpcAMPs $(100 \mu \mathrm{M}$, Enzo Life Sciences, UK) and PKI (10 $\mu \mathrm{M}$, Calbiochem, UK). Cells were incubated with inhibitors for $24 \mathrm{~h}$.

\section{Plasmids, oligonucleotides and RT-PCR}

A cDNA encoding human Wnt-11 (GeneCopoeia, Frederick, MD) was subcloned into pcDNA3.1 (Invitrogen) using EcoRI and XhoI. Plasmids expressing control and WNT11 shRNAs were generated by ligation of annealed oligonucleotides into pTER (21) with 5' - 3' sequences as follows:

Control: GATCCCCTTCTCCGAACGTGTCACGTTTCAAGAGAACGTGACACGTTCGG

AGAATTTTTGGAAA

WNT11: GATCCCCGGACTCGGAACTCGTCTATTTCAAGAGAATAGACGAGTTCCG AGTCCTTTTTGGAAA

For RT-PCR, total RNA was extracted and analysed according to manufacturer's instructions (One step RTPCR, Qiagen, UK). The primer sequences (5' - 3') were:

WNT11 - CGATGCTCCTATGAAGGTGAAA, CTTCCGTTGGATGTCTTGTTG

NSE - CTCATCAGCTCAGGTCTCTC, CCTTACACACGGCCAGAGAC

GAPDH - TGTTGCCATCAATGACCCCTT, CTCCACGACGTACTCAGCG

The primers for $A S C L 1$ were as previously described [17]. The number of cycles used (32 for WNT11 and NSE, 40 for ASCL1 and 22 for GAPDH) was determined empirically so that there was a linear relationship between target quantity and product yield to permit semi-quantitative analysis. Analysis by real-time q-PCR was done using iTaq SYBR Green premix (BIO-RAD) and the following conditions: $95^{\circ} \mathrm{C}$ for $10 \mathrm{~min}, 40$ cycles at $95^{\circ} \mathrm{C}$ for $15 \mathrm{~s}, 60^{\circ} \mathrm{C}$ for $1 \mathrm{~min}$ and a dissociation stage $\left(95^{\circ} \mathrm{C}\right.$ for $15 \mathrm{~s}, 60^{\circ} \mathrm{C}$ for $1 \mathrm{~min}, 95^{\circ} \mathrm{C}$ for $15 \mathrm{~s}, 60^{\circ} \mathrm{C}$ for $15 \mathrm{sec})$. GAPDH and ASCL1 primers used were as previously described [17]. Relative levels of mRNA expression were calculated using the Comparative CT Method, $2^{-\Delta \Delta C T}(\Delta \Delta C T: \Delta C T$ sample $-\Delta C T$ calibrator) [29]. Experiments were performed in triplicate and statistical significance was analysed using Student's t-Test, comparing $\triangle \mathrm{CT}$ (CT target - CT housekeeping) obtained from the calibrator.

\section{Transfections}

For Wnt-11 overexpression, LNCaP and RWPE-1 cells were transiently transfected with pcDNA Wnt-11, empty vector or pEGFP-C1 (Clontech) using FugeneHD (Roche) and assayed $48 \mathrm{~h}$ later. PKA inhibitors were added 24 hours after transfection. To generate LNCaP cell lines expressing control and WNT11 shRNAs, LNCaP/TR2 cells [30] were transfected with pTER control shRNA or WNT11 shRNA plasmids using Tfx-50 (Promega), selected in medium containing $6 \mu \mathrm{g} / \mathrm{ml}$ blasticidin and $300 \mu \mathrm{g} / \mathrm{ml}$ Zeocin (Invitrogen, UK) and colonies then picked and expanded. WNT11 expression was determined by RT-PCR analysis of clones cultured for 3 days in androgen-depleted medium. Three WNT11 shRNA clones with reduced expression of Wnt-11 and three control shRNA clones were selected and used for experiments. Experiments were conducted in medium containing 5\% CSS and doxycycline to induce expression of the shRNA. Despite the use of a Tet-inducible LNCaP cell line and the pTER vector, expression of WNT11 shRNA in the expanded clones was only weakly affected by doxycycline (unpublished observations). siRNA transfections were performed according to the manufacturer's protocols using DharmaFECT 2 (Dharmacon, USA). PC3 cells were seeded in 6-well plates for $48 \mathrm{~h}$ before transfection using ON-TARGETplus SMARTpool WNT11 siRNA or ON-TARGETplus nontargeting pool (Dharmacon, USA). Transfected cells were processed for RNA extraction (after 24 and $48 \mathrm{~h}$ ) or caspase activity (after 24, 48 and $72 \mathrm{~h}$ ) or used in cell migration assays (72 h).

\section{Antibodies, western blotting and immunostaining}

The following antibodies were used: mouse anti-NSE (BBS/NC/V1-H14, Dako), rabbit anti-cleaved caspase-9 (Asp330; 9501) and rabbit anti-cleaved PARP (Asp214; 9541) (both from Cell Signalling Technology), rabbit anti-AR (N20; sc-816, Santa Cruz Biotechnology), mouse anti-HSP60 (SPA-806, Stressgen) and goat antiWnt-11 (R\&D systems, AF2647). Rabbit anti-Wnt-11 
(H-95; sc-50360, Santa Cruz Biotechnology) was also used for immunohistochemistry to confirm the results using goat anti-Wnt-11. For immunocytochemistry, transfected RWPE-1 and LNCaP cells were fixed in 4\% paraformaldehyde for $15 \mathrm{~min}$, blocked with 5\% BSA/PBS for $30 \mathrm{~min}$ and incubated with rabbit anti-Wnt-11 (1:20) and/or mouse anti-AR (clone AR441, Dako, 1:40) overnight at $4^{\circ} \mathrm{C}$. Alexa Fluor 488 goat anti-mouse IgG $(\mathrm{H}+\mathrm{L})$ and/or Alexa Fluor 555 goat anti-rabbit IgG $(\mathrm{H}+\mathrm{L})$ (Invitrogen) were used as secondary antibodies (1:500, $60 \mathrm{~min}$ at room temperature) and nuclei were counterstained using 1.0 $\mu \mathrm{M}$ TO-PRO-3 (Invitrogen). Images were acquired on a LSM 510 laser scanning confocal microscope (Carl Zeiss). Preparation of extracts and western blotting were done as previously described (23). Blots were stripped and re-probed for HSP60 to control for gel loading. Human Prostate cancer and adjacent normal prostate tissues (tissue arrays and single tissue slides) were purchased from SuperBioChips Laboratories (single slides and TMA-CA3), Stretton Scientific (TMA-A222) and US BioMax Inc. (TMA-PR951). Samples were provided as formalin-fixed, paraffin-embedded and sectioned for histological analysis. Representative haematoxylineosin-stained sections were examined to evaluate the histopathological characteristics of the lesion to be analysed. The suppliers provided patient pathology data, when available. All samples were independently analysed for Gleason grade by a pathologist (MMW). Sections were deparaffinised and rehydrated using graded alcohol concentrations. Antigen retrieval was performed by incubation in $10 \mathrm{mM}$ sodium citrate $\mathrm{pH} 6.0$ and heating in a microwave oven at $560 \mathrm{~W}$ for $8 \mathrm{~min}$. Endogenous peroxidase was quenched using $3 \% \mathrm{H}_{2} \mathrm{O}_{2}$ for $30 \mathrm{~min}$. Following blocking (30 min in PBS with 1.5\% horse serum), sections were incubated with goat anti-Wnt-11 at 1:200, rabbit anti-Wnt-11 at 1:50 or anti-AR at 1:200 for $1 \mathrm{~h}$ at room temperature. Washing and antibody visualisation was done using the Vectastain Elite ABC Standard kit according to manufacturer's instructions (Vector Labs). TMA analysis was performed using an image analyser (ChromaVision ACIS II, Zeiss, Germany). Tissue samples were scored by MMW for Wnt-11 levels in prostate cancer epithelial cells $(0=$ no signal; $1=$ positive, and $2=$ strongly positive).

\section{Assays for morphology, apoptosis, cell number, migration and invasion}

For morphological analysis, images of live cells were taken and analysed using ImageJ software http://rsb.info. nih.gov/ij. The numbers of cells with and without neurites longer than two cell bodies were counted in photomicrographs of control and WNT11 shRNA cells that had been cultured for 4 days in hormone-depleted medium. The data presented are the means derived from counting three fields of 100 cells from a representative experiment $(n=2)$. For caspase assays, trypsinised cells were plated at a density of 5000 cells per well in opaque 96-well cell culture plates (Perkin Elmer). At the indicated times after plating, an equal volume of CaspaseGlo 3/7 reagent (Promega) was added, the plate was incubated for $30 \mathrm{~min}$ and readings were taken using a luminometer. To normalise for cell number, MTT assays were conducted in parallel using the same number of cells plated in normal 96-well tissue culture plates. The number of viable cells was measured by adding $200 \mu \mathrm{l}$ sterile MTT (3- [4,5-dimethylthiazol-2-yl]2,5-diphenyltetrazolium bromide, $5 \mathrm{mg} / \mathrm{ml}$ ) diluted in fresh medium to each well, loosely wrapping the plate in foil and incubating at $37^{\circ} \mathrm{C}$ for $3 \mathrm{~h}$. The media were then replaced with $250 \mu \mathrm{l}$ DMSO, followed by $25 \mu \mathrm{l}$ glycine buffer (0.1 M glycine, $0.1 \mathrm{M} \mathrm{NaCl}, \mathrm{pH} 10.5)$ and absorbance at $570 \mathrm{~nm}$ was read within $10 \mathrm{~min}$. Details of PC3 cell migration assays were as described previously (24). Briefly, cells were trypsinised and plated at $2 \times 10^{4}$ cells/well onto 12 -micron pore Transwell filters with a polycarbonate membrane (Corning). Following 6 $\mathrm{h}$ incubation, the MTT assays were used to determine the number of migrated cells and the results were plotted as Migration Index (Mi), which is the percentage of migrated cells compared to the total number of cells seeded. MTT assays were used to confirm that that there was no effect on total cell number during the $6 \mathrm{~h}$ time course of the experiments. LNCaP invasion assays were done in similar manner to Vias et al. [21], except that LNCaP cells were first transfected with vector or Wnt-11 plasmid and, after 48 hours, $5 \times 10^{5}$ transfected cells were plated on Matrigel-coated Transwell filters (BD Biosciences) in growth medium containing 1\% FCS or $1 \%$ CSS supplemented with DHT to prevent expression of endogenous Wnt-11 (both conditions gave similar results). After a further $42 \mathrm{~h}$, the number of invaded cells was determined by MTT assay. The total number of cells plated was also determined by MTT assay.

\section{Data analysis}

All data were analysed as means \pm standard deviation. Statistical significance was determined using Student's t-test or chi-squared test, as appropriate; results were considered significant for $\mathrm{p}<0.05$

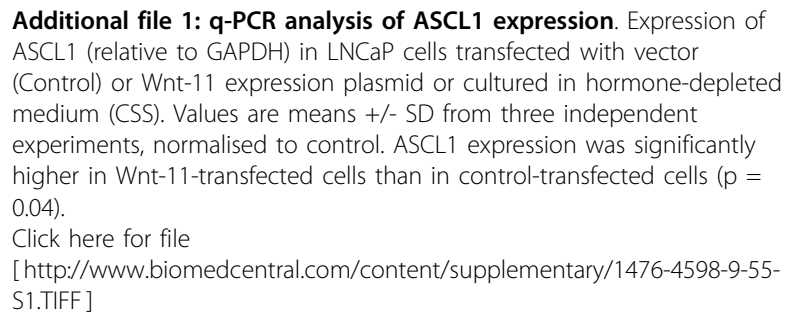


Additional file 2: Comparison of transfection efficiency of LNCaP and RWPE-1 cells. (a, b) Images of LNCaP (a) and RWPE-1 (b) cells transfected for $48 \mathrm{~h}$ with GFP plasmid. GFP is shown in green and cell nuclei in blue. A similar proportion of cells (5\%) expressed GFP in both cell lines. (c, d) LNCaP (c) and RWPE-1 (d) were transfected with Wnt-11 plasmid and, after $48 \mathrm{~h}$, stained for Wnt-11 (red). Wnt-11 staining was of a similar intensity in both cell lines.

Click here for file

[ http://www.biomedcentral.com/content/supplementary/1476-4598-9-55S2.TIFF ]

\section{Acknowledgements}

We thank Maria Vivanco (CIC bioGUNE) for critical reading of the manuscript. Funded by the Prostate Cancer Charity UK, the Garfield Weston Foundation and Cancer Research UK (RK and JW), the Spanish Ministry of Education and Science (SAF 2005-06122 and SAF 2008-00768), the Departments of Industry, Tourism and Trade (Etortek) and Department of Innovation Technology of the Government of the Autonomous Community of the Basque Country (RK).

\section{Author details}

'Department of Oncology, Imperial College London, London, UK.

${ }^{2}$ Department of Histopathology, Imperial College London, London, UK.

${ }^{3}$ Centre for Cooperative Research in Biosciences (CIC bioGUNE), Derio, Spain.

\section{Authors' contributions}

PUO participated in the design of the study, acquired the majority of the data, analysed and interpreted data and drafted the manuscript; YK and MC acquired, analysed and interpreted data; MMW analysed and interpreted immunohistochemistry data and helped draft the manuscript; SD and RSD participated in the interpretation of data; JW participated in the study design and helped draft the manuscript; RMK conceived, designed and coordinated the study, acquired, analysed and interpreted data and drafted the manuscript. All authors read and approved the final manuscript.

\section{Author's information}

SD's present address is UCL Cancer Institute, University College London. Correspondence for RM should be sent to r.kypta@ic.ac.uk or rkypta@cicbiogune.es.

\section{Competing interests}

The authors declare that they have no competing interests.

Received: 19 November 2009 Accepted: 10 March 2010 Published: 10 March 2010

\section{References}

1. Klaus A, Birchmeier W: Wnt signalling and its impact on development and cancer. Nat Rev Cancer 2008, 8:387-398.

2. Zhu H, Mazor M, Kawano Y, Walker MM, Leung HY, Armstrong K, Waxman J, Kypta RM: Analysis of Wnt gene expression in prostate cancer: mutual inhibition by WNT11 and the androgen receptor. Cancer Res 2004, 64:7918-7926.

3. Tada M, Concha ML, Heisenberg CP: Non-canonical Wnt signalling and regulation of gastrulation movements. Semin Cell Dev Biol 2002, 13:251-260.

4. Majumdar A, Vainio S, Kispert A, McMahon J, McMahon AP: Wnt11 and Ret/Gdnf pathways cooperate in regulating ureteric branching during metanephric kidney development. Development 2003, 130:3175-3185.

5. Eisenberg CA, Eisenberg LM: WNT11 promotes cardiac tissue formation of early mesoderm. Dev Dyn 1999, 216:45-58.

6. Ouko L, Ziegler TR, Gu LH, Eisenberg LM, Yang WW: Wnt11 signaling promotes proliferation, transformation, and migration of IEC6 intestinal epithelial cells. J Biol Chem 2004, 279:26707-26715.

7. Lin Z, Reierstad S, Huang CC, Bulun SE: Novel estrogen receptor-alpha binding sites and estradiol target genes identified by chromatin immunoprecipitation cloning in breast cancer. Cancer Res 2007, 67:5017-5024.

8. Railo A, Nagy II, Kilpelainen P, Vainio S: Wnt-11 signaling leads to downregulation of the Wnt/beta-catenin, JNK/AP-1 and NF-kappaB pathways and promotes viability in the CHO-K1 cells. Exp Cell Res 2008, 314:2389-2399.

9. Flaherty MP, Abdel-Latif A, Li Q, Hunt G, Ranjan S, Ou Q, Tang XL, Johnson RK, Bolli R, Dawn B: Noncanonical Wnt11 signaling is sufficient to induce cardiomyogenic differentiation in unfractionated bone marrow mononuclear cells. Circulation 2008, 117:2241-2252.

10. Zhou W, Lin L, Majumdar A, Li X, Zhang X, Liu W, Etheridge L, Shi Y, Martin J, Ven Van de $W$, et al: Modulation of morphogenesis by noncanonical Wnt signaling requires ATF/CREB family-mediated transcriptional activation of TGFbeta2. Nat Genet 2007, 39:1225-1234.

11. Maye P, Zheng J, Li L, Wu D: Multiple mechanisms for Wnt11-mediated repression of the canonical Wnt signaling pathway. J Biol Chem 2004, 279:24659-24665.

12. Cindolo $L$, Cantile M, Vacherot $F$, Terry $S$, de la Taille A: Neuroendocrine differentiation in prostate cancer: from lab to bedside. Urol Int 2007, 79:287-296.

13. Yuan TC, Veeramani S, Lin MF: Neuroendocrine-like prostate cancer cells: neuroendocrine transdifferentiation of prostate adenocarcinoma cells. Endocr Relat Cancer 2007, 14:531-547.

14. Deeble PD, Cox ME, Frierson HF Jr, Sikes RA, Palmer JB, Davidson RJ, Casarez EV, Amorino GP, Parsons SJ: Androgen-independent growth and tumorigenesis of prostate cancer cells are enhanced by the presence of PKA-differentiated neuroendocrine cells. Cancer Res 2007, 67:3663-3672.

15. Cox ME, Deeble PD, Lakhani S, Parsons SJ: Acquisition of neuroendocrine characteristics by prostate tumor cells is reversible: implications for prostate cancer progression. Cancer Res 1999, 59:3821-3830.

16. Burchardt T, Burchardt M, Chen MW, Cao Y, de la Taille A, Shabsigh A, Hayek O, Dorai T, Buttyan R: Transdifferentiation of prostate cancer cells to a neuroendocrine cell phenotype in vitro and in vivo. J Urol 1999, 162:1800-1805

17. Vias M, Massie CE, East P, Scott H, Warren A, Zhou Z, Nikitin AY, Neal DE, Mills IG: Pro-neural transcription factors as cancer markers. BMC Med Genomics 2008, 1:17.

18. Litvinov IV, Griend Vander DJ, Xu Y, Antony L, Dalrymple SL, Isaacs JT: Lowcalcium serum-free defined medium selects for growth of normal prostatic epithelial stem cells. Cancer Res 2006, 66:8598-8607.

19. Tada M, Smith JC: Xwnt11 is a target of Xenopus Brachyury: regulation of gastrulation movements via Dishevelled, but not through the canonical Wnt pathway. Development 2000, 127:2227-2238.

20. Heisenberg CP, Tada M, Rauch GJ, Saude L, Concha ML, Geisler R, Stemple DL, Smith JC, Wilson SW: Silberblick/Wnt11 mediates convergent extension movements during zebrafish gastrulation. Nature 2000, 405:76-81.

21. Vias M, Burtt G, Culig Z, Veerakumarasivam A, Neal DE, Mills IG: A role for neurotensin in bicalutamide resistant prostate cancer cells. Prostate 2007 67:190-202.

22. Vanoverberghe $K$, Abeele Vanden $F$, Mariot $P$, Lepage $G$, Roudbaraki $M$, Bonnal JL, Mauroy B, Shuba Y, Skryma R, Prevarskaya N: Ca2+ homeostasis and apoptotic resistance of neuroendocrine-differentiated prostate cancer cells. Cell Death Differ 2004, 11:321-330.

23. Eisenberg CA, Gourdie RG, Eisenberg LM: Wnt-11 is expressed in early avian mesoderm and required for the differentiation of the quail mesoderm cell line QCE-6. Development 1997, 124:525-536.

24. Terami H, Hidaka K, Katsumata T, lio A, Morisaki T: Wnt11 facilitates embryonic stem cell differentiation to Nkx2.5-positive cardiomyocytes. Biochem Biophys Res Commun 2004, 325:968-975.

25. Koyanagi M, Haendeler J, Badorff C, Brandes RP, Hoffmann J, Pandur P, Zeiher AM, Kuhl M, Dimmeler S: Non-canonical Wnt signaling enhances differentiation of human circulating progenitor cells to cardiomyogenic cells. J Biol Chem 2005, 280:16838-16842.

26. Deng X, Liu H, Huang J, Cheng L, Keller ET, Parsons SJ, Hu CD: lonizing radiation induces prostate cancer neuroendocrine differentiation through interplay of CREB and ATF2: implications for disease progression. Cancer Res 2008, 68:9663-9670. 
27. Komiya A, Suzuki H, Imamoto T, Kamiya N, Nihei N, Naya Y, Ichikawa T, Fuse $\mathrm{H}$ : Neuroendocrine differentiation in the progression of prostate cancer. Int J Urol 2009, 16:37-44.

28. Griend Vander DJ, Karthaus WL, Dalrymple S, Meeker A, DeMarzo AM, Isaacs JT: The role of CD133 in normal human prostate stem cells and malignant cancer-initiating cells. Cancer Res 2008, 68:9703-9711.

29. Pfaffl MW: A new mathematical model for relative quantification in realtime RT-PCR. Nucleic Acids Res 2001, 29:e45.

30. Kawano Y, Kitaoka M, Hamada Y, Walker MM, Waxman J, Kypta RM: Regulation of prostate cell growth and morphogenesis by Dickkopf-3. Oncogene 2006, 25:6528-6537.

doi:10.1186/1476-4598-9-55

Cite this article as: Uysal-Onganer et al:: Wnt-11 promotes

neuroendocrine-like differentiation, survival and migration of prostate cancer cells. Molecular Cancer 2010 9:55.

\section{Submit your next manuscript to BioMed Central} and take full advantage of:

- Convenient online submission

- Thorough peer review

- No space constraints or color figure charges

- Immediate publication on acceptance

- Inclusion in PubMed, CAS, Scopus and Google Scholar

- Research which is freely available for redistribution

Submit your manuscript at www.biomedcentral.com/submit 